\title{
Profiling of external metabolites during production of hantavirus nucleocapsid protein with recombinant Saccharomyces cerevisiae
}

\author{
Linas Antoniukas · Hartmut Grammel · \\ Kestutis Sasnauskas · Udo Reichl
}

Received: 31 August 2007/Accepted: 9 October 2007/Published online: 6 November 2007

(C) Springer Science+Business Media B.V. 2007

\begin{abstract}
Recombinant strains of Saccharomyces cerevisiae, producing hantavirus Puumala nucleocapsid protein for diagnostics and as a candidate vaccine were analyzed for uptake and excretion of intermediary metabolites during process optimization studies of fed-batch bioreactor cultures. Concentrations of glucose, maltose, galactose, pyruvate, acetaldehyde, ethanol, acetate, succinate and formaldehyde (used as a selection agent) were measured in the culture medium in order to find a metabolite pattern, indicative for the physiological state of the producer culture. When the inducer galactose was employed as a growth substrate, the metabolite profile of recombinant yeast cells was different from those of the non-recombinant original strain which excreted considerable amounts of metabolites with this substrate. In contrast, galactose-induced heterologous gene expression was indicated by the absence of excreted intermediary metabolites, except succinate. A model strain expressing a GFP fusion of hantavirus
\end{abstract}

L. Antoniukas · H. Grammel · U. Reichl

Max-Planck-Institute for Dynamics of Complex Technical Systems, Sandtorstr. 1, 39106 Magdeburg, Germany

L. Antoniukas $(\bowtie) \cdot K$. Sasnauskas

Institute of Biotechnology, V. Graiciuno 8, 02241 Vilnius, Lithuania

e-mail: linasa@ibt.lt

U. Reichl

Otto-von-Guericke University, Universitätsplatz 2,

39106 Magdeburg, Germany nucleocapsid protein differed in the excretion of metabolites from strains without GFP. In addition, the influence of alkali ions, employed for $\mathrm{pH}$ control is also demonstrated.

Keywords Fed-batch cultivation ·

Hantavirus - Metabolite profiling ·

Saccharomyces cerevisiae

\section{Introduction}

The necessity for a further improvement of the production of vaccines against hantavirus is strikingly demonstrated by very recent data of a dramatic increase in the number of infections in Europe (e.g., Koch et al. 2007). We have recently developed a process for producing hantavirus Puumala nucleocapsid protein ( $\mathrm{N}$ protein) for diagnostics and as a candidate vaccine (Dargeviciute et al. 2002) using Saccharomyces cerevisiae as a recombinant host (Antoniukas et al. 2006). The major hallmark of our process is the development of a malt extract-enriched YNB (YNB-M) medium in combination with a galactose-inducible expression system, which is activated by feeding concentrated YNB-M plus galactose at a constant rate for a $3 \mathrm{~h}$ period. The growth characteristics of the fed-batch cultures are presented in Antoniukas et al. (2006).

Yeasts, and particularly Saccharomyces cerevisiae, are widely used as a host organism for the production 
of recombinant pharmaceutical proteins and enzymes for food industry. However, the high-level expression of a foreign protein especially under the control of strong promoters results in shifts of metabolite fluxes towards the desired product, thus accelerating the overall metabolic burden (Janes et al. 1990; Snoep et al. 1995). One analytically accessible indication of imbalances of central metabolic pathways, either due to environmental factors or provoked by the heterologous expression vector, is the assessment of metabolites originating from central metabolism, which are excreted into the culture broth. Generally, the metabolic products of yeast cells are attributed to two effects of carbohydrate catabolism: (i) formation of fermentation products for the regeneration of redox cofactors under limiting oxygen concentrations. The phenomenon that in case of glucose excess, even though oxygen may be present, fermentation predominates over respiration, has been known as the Crabtree effect. (ii) Alternatively, the occurrence of metabolic products in culture supernatants, predominantly during oxidative growth has been interpreted in terms of "overflow metabolism" where metabolic bottlenecks between high and low capacity pathways lead to the accumulation of metabolites which are disposed by export to the environment.

In the present study we have compared the metabolic profiles of a hantavirus $\mathrm{N}$ protein producing recombinant $S$. cerevisiae and the nonrecombinant original strain in bioreactor fed-batch cultivations. We address the question whether monitoring of excreted metabolites allows the assessment of the current metabolic state of the producer culture, which may be valuable for the control and optimization of the process.

\section{Materials and methods}

Microorganisms and growth conditions

The recombinant $S$. cerevisiae strains FH4C[pFD36his-N-GFP] (Antoniukas et al. 2006) and FH4C [pFX7-6his-N] (Dargeviciute et al. 2002) were used for the intracellular production of the recombinant hexahistidine tagged $\mathrm{N}$ protein variants 6his-N-GFP and 6his-N, respectively. The employed expression vector pFD3 was described previously (Sasnauskas et al. 1999). All media used in this study were described in Antoniukas et al. (2006)

\section{Bioreactor fed-batch cultivations}

The cultivation strategy, bioreactor equipment and experimental setup were described previously in detail (Antoniukas et al. 2006). Briefly, several parameters were controlled during the cultivation run: airflow was set constant at $21 \mathrm{~min}^{-1}\left(1 \mathrm{v} / \mathrm{v} \mathrm{min}{ }^{-1}\right)$, temperature was maintained at $30^{\circ} \mathrm{C}$, and $\mathrm{pH}$ was controlled at 5 , through the addition of either $1 \mathrm{M} \mathrm{NaOH}, 1 \mathrm{M} \mathrm{KOH}$, or $10 \%(\mathrm{w} / \mathrm{v}) \mathrm{NH}_{4} \mathrm{OH}$ and $1 \mathrm{M} \mathrm{HCl}$. The dissolved $\mathrm{O}_{2}$ concentration $\left(\mathrm{pO}_{2}\right)$ was maintained at $70 \%$ of air saturation through automatic variation of the agitation speed. The bioreactor vessel was filled with 21 of filtersterilized initial medium. After the inoculation, formaldehyde was added (final concentration $0.225 \mathrm{~g} \mathrm{l}^{-1}$ ) to give a selective pressure, due to a formaldehyde dehydrogenase gene of the expression vector. By the end of the initial batch phase, galactose was added as inducer of recombinant gene expression by a transient feeding of $250 \mathrm{ml} 8 \times$ YNB-galactose medium at a constant rate.

Detection and quantification of recombinant proteins

The recombinant 6 his-N-GFP protein was detected by measuring GFP fluorescence intensity in yeast cells with a fluorescence spectrophotometer as described previously (Antoniukas et al. 2006).

Analysis of metabolites

Concentrations of glucose, maltose, galactose, ethanol, acetaldehyde, acetate and succinate in culture medium were measured with enzymatic test kits (R-Biopharm, Germany). Pyruvate concentrations were measured by HPLC equipped with a photodiode array detector. Separation was carried out isocratically using an Inertsil ODS $5 \mu$ column and $\mathrm{NH}_{4} \mathrm{HPO}_{4}(0.1 \mathrm{M}), \mathrm{pH}=2.6$ as eluent. Metabolite yield coefficients $\left(\mathrm{Y}_{\mathrm{P} / \mathrm{S}}\right)$ were calculated using concentration data points from exponential grow phases. 


\section{Results and discussion}

Metabolite profiles of fed-batch cultures with non-producing and hantavirus $\mathrm{N}$ protein producing $S$. cerevisiae strains

Extracellular metabolite profiles of cultivations with the original non-producing wild type strain $S$. cerevisiae $\mathrm{FH} 4 \mathrm{C}$ and the recombinant producer strains S. cerevisiae $\mathrm{FH} 4 \mathrm{C}[\mathrm{pFX} 7-\mathrm{N}]$, S. cerevisiae $\mathrm{FH} 4 \mathrm{C}$ [pFX7-6his-N] and S. cerevisiae FH4C[pFD3-6his$\mathrm{N}-\mathrm{GFP}]$, the latter expressing a $\mathrm{N}$ protein-GFP fusion for monitoring heterologous gene expression were examined (for a detailed description of employed strains, media and cultivation conditions, see Antoniukas et al. (2006)). Figure 1 shows fed-batch cultivations of the wild type (Fig. 1a) and the 6his-
N-GFP expressing strain (Fig. 1b). With all strains employed, fed-batch cultivations in a 51 bioreactor could be decomposed into three distinct cultivation phases, each represented by a specific metabolite profile in the culture supernatant. $\mathrm{NH}_{4} \mathrm{OH}$ was used for $\mathrm{pH}$ control during these cultivations.

\section{Glucose/maltose growth phase}

When looking at the non-recombinant control strain S. cerevisiae $\mathrm{FH} 4 \mathrm{C}$, the initial diauxic growth phase with maltose (originating from the malt extract supplement of YNB medium) and glucose lasted from 0 to $10 \mathrm{~h}$ (Fig. 1a). During this period fermentative pathways were induced, as indicated by the release of ethanol, as well as pyruvate, and

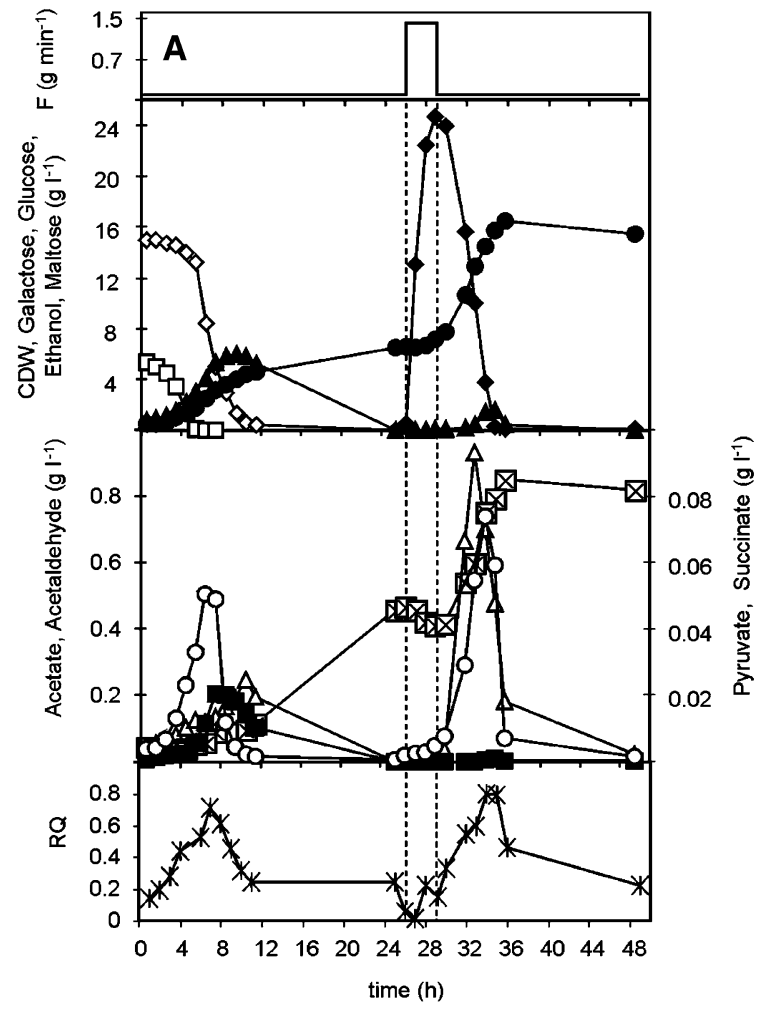

Fig. 1 Substrate feed rate (F) and profiles of biomass growth $(\boldsymbol{\bullet})$, glucose $(\square)$, maltose $(\diamond)$, ethanol $(\mathbf{\Delta})$, galactose $(\diamond)$, fluorescence intensity of the heterologous 6his-N-GFP $(\nabla)$, formaldehyde $(\times)$ (was added as selective agent), pyruvate $(0)$, acetaldehyde $(\boldsymbol{\square})$, acetate $(\Delta)$ and succinate $(\boldsymbol{\nabla})$ during the fedbatch cultivation with $S$. cerevisiae $\mathrm{FH} 4 \mathrm{C}$ (a) and recombinant producer strain S. cerevisiae FH4C[pFD3-6his-N-GFP] (b). Metabolites were determined with enzymatic test kits (R-Biopharm.

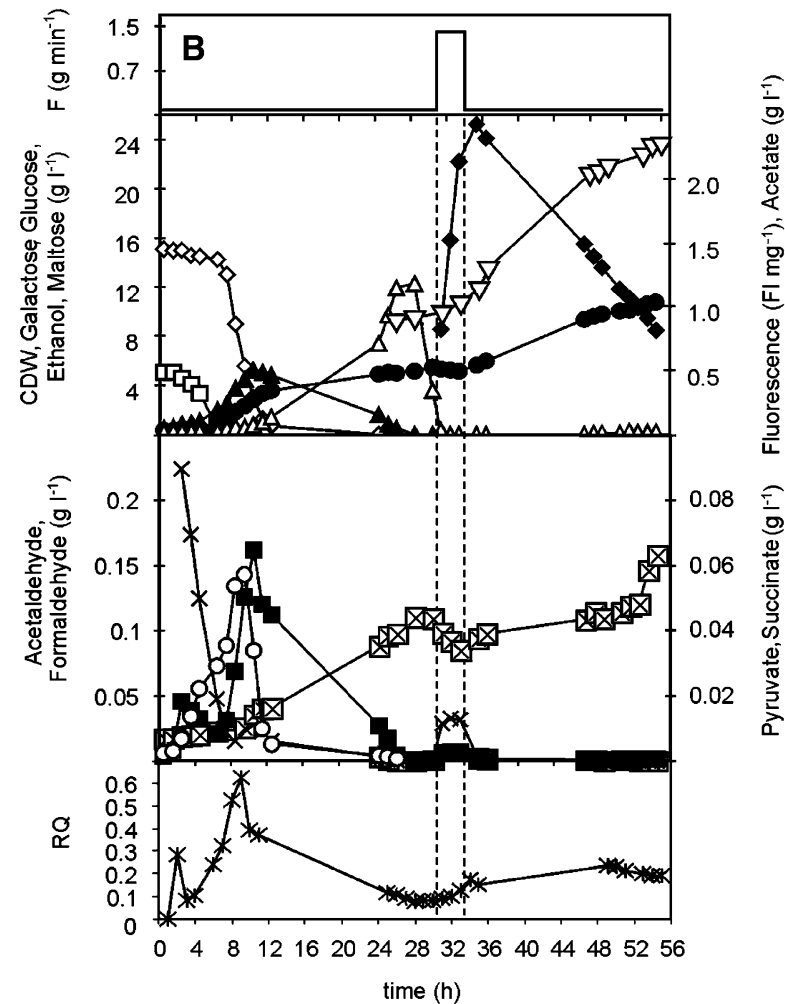

Germany). The feeding phase of concentrated medium with galactose is indicated by dashed lines. RQ $=Q_{\mathrm{CO}_{2}} / Q_{\mathrm{O}_{2}}$, with $Q_{\mathrm{O}_{2}}=\dot{V}_{\mathrm{G}}\left(Y_{\mathrm{O}_{2}}^{\alpha}-Y_{\mathrm{O}_{2}}^{\omega}\right) /\left(V_{\mathrm{F}} \bullet 22.4(l / m o l)\right) ; \dot{V}_{\mathrm{G}}$ : Vol. gas phase; $V_{\mathrm{F}}$ : Vol. bioreactor; $Y_{\mathrm{O}_{2}} \alpha, \omega: \mathrm{O}_{2}$ concentration, determined by gas chromatography (Agilent Mikro-GC) at the bioreactor inlet and outlet, respectively $Q_{\mathrm{CO}_{2}}$ was calculated with $Q_{\mathrm{CO}_{2}}=$ $\dot{V}_{\mathrm{G}}\left(Y_{\mathrm{CO}_{2}}^{\omega}-Y_{\mathrm{CO}_{2}}^{\alpha}\right) /\left(V_{\mathrm{F}} \bullet 22.4(l / m o l)\right)$ 
acetaldehyde as minor products. Thus, growth was "respiro-fermentative", a well known mixed metabolic mode when $S$. cerevisiae is cultivated with excess of glucose. As the concentrations of glucose and maltose were decreasing at a later stage of this growth phase, the cells were relieved from glucose repression and the reconsumption of the formerly excreted components was initiated. We also found significant amounts of succinate in the supernatants throughout the cultivation (see below). The accumulation of acetate, acetaldehyde and pyruvate during growth with glucose, was also indicated by onlinegas analysis and the respiratory quotient (RQ) was rising to 0.7 during that growth period. No significant differences between the original strain and the producer strains were observed during this period, as expected because of the absence of the inductor galactose.

\section{Ethanol growth phase}

This phase was characterized by the exclusively respiratory consumption of ethanol (but also pyruvate and acetaldehyde). RQ values were decreasing and stayed at a constant level of about 0.2 , which is a characteristic low value for the oxidation of ethanol. Interestingly, when examining metabolites in the culture medium, we observed a striking difference here, between the model strain which carries a $\mathrm{N}$ protein-GFP fusion and strains with either no vector (wild type) or expressing a construct ( $\mathrm{N}$ protein or 6his-N protein) without GFP. The GFP fusion strain excreted considerable amounts of acetate during growth with ethanol $\left(>1 \mathrm{~g} \mathrm{l}^{-1}\right.$, Fig. 1b), whereas GFP-free strains produced no acetate but instead reconsumed the same compound, which was excreted during the preceding glucose/maltose growth phase (Table 1). This finding clearly limits the application of GFP strains for process development and optimization, at least for the hantavirus $\mathrm{N}$ protein expression system considered here. However, an impact of GFP expression on cellular physiology has been observed recently also in bacterial cells (Allison and Sattenstall 2007). No differences between GFP expressing strains and GFP free strains were observed with respect to growth rates and biomass yields. Note, that a basal expression of GFP could be detected even before galactose was added as inducer for recombinant gene expression, when $G A L$ genes were relieved from glucose repression (Fig. 1b). In the present case, acetate production may constitute a symptom for unbalanced growth with high metabolic fluxes through the alcohol dehydrogenase and acetaldehyde dehydrogenase reactions, which during growth with ethanol have to provide the complete supply of reducing equivalents (NADH and NADPH) for oxidative phosphorylation and biosyntheses, respectively. The basal constitutive expression of the recombinant GFP fusion protein, but not expression of the GFP-free N protein, might increase the demand for $\mathrm{NADPH}$, accompanied by the excretion of acetate.

\section{Galactose growth phase (hantavirus $N$ protein production phase)}

The metabolic profiles after feeding of galactose, indicate that growth was respiratory as ethanol concentration was always below the detection limit. In the wild type strain S. cerevisiae FH4C the RQ increased again to 0.8 which is close to the theoretical value $(R Q=1)$ for the oxidation of galactose. The galactose growth phase was characterized by the transient accumulation of acetate and pyruvate, which both exhibited sharp peak-like kinetics (Fig. 1a). We interpret this finding as a form of overflow metabolism of intermediates when the assimilatory capabilities (e.g., amino acid synthesis) cannot cope with the high catabolic fluxes through the glycolytic pathway. Strikingly, the recombinant producer strains S. cerevisiae FH4C[pFX7-6his-N] and S. cerevisiae FH4C[pFD3-6his-N-GFP] showed a different pattern and neither pyruvate nor acetate could be detected during growth with galactose under the growth conditions applied here (YNB-M medium, $\mathrm{NH}_{4} \mathrm{OH}$ $\mathrm{pH}$ control, Fig. 1b and Table 1). Correspondingly, we could not observe an increase of the RQ. This finding is presumably caused by a reduced growth rate of the producer compared to the wild-type strain thus preventing overflow metabolism.

In contrast to the ethanol growth phase, the metabolic profile of the producer strains $S$. cerevisiae FH4C[pFX7-6his-N] and S. cerevisiae FH4C[pFX7$\mathrm{N}$ ] was basically identical to the GFP fusion proteinexpressing strain S. cerevisiae FH4C[pFD3-6his-NGFP] after galactose induction of recombinant gene expression (Table 1). 
Table 1 Yield coefficients of extracellular metabolites from fed-batch cultivations of S. cerevisiae in the bioreactor

\begin{tabular}{|c|c|c|c|c|c|c|}
\hline \multirow[t]{2}{*}{ Cultivation } & \multirow[t]{2}{*}{ Growth substrate } & \multicolumn{5}{|c|}{$Y_{\mathrm{P} / \mathrm{S}}\left(\mathrm{mg} \mathrm{g}^{-1}\right)$} \\
\hline & & Pyruvate & Ethanol & Acetaldehyde & Acetate & Succinate \\
\hline \multirow{3}{*}{$\begin{array}{l}\text { S. cerevisiae } \mathrm{FH} 4 \mathrm{C}[\mathrm{pFD} 3-6 \text { his-N- } \\
\text { GFP] YNB medium, pH control } \\
\text { with } \mathrm{NaOH}\end{array}$} & Glucose & 7 & 454 & 4 & 11 & 0 \\
\hline & Ethanol & 0 & - & 0 & 268 & 0 \\
\hline & Galactose & 2 & 227 & 9 & 57 & 0 \\
\hline \multirow{3}{*}{$\begin{array}{l}\text { S. cerevisiae } \mathrm{FH} 4 \mathrm{C}[\mathrm{pFD} 3-6 \text { his-N- } \\
\text { GFP] YNB-M medium, pH } \\
\text { control with } \mathrm{NH}_{4} \mathrm{OH}\end{array}$} & Glucose \& Maltose & 4 & 284 & 8 & 7 & 0 \\
\hline & Ethanol & 0 & - & 0 & 388 & 5 \\
\hline & Galactose & 0 & 3 & 0 & 0 & 1 \\
\hline \multirow{3}{*}{$\begin{array}{l}\text { S. cerevisiae } \mathrm{FH} 4 \mathrm{C}[\mathrm{pFX} 7-6 \text { his-N] } \\
\text { YNB-M medium, pH control } \\
\text { with } \mathrm{NH}_{4} \mathrm{OH}\end{array}$} & Glucose \& Maltose & 3 & 301 & 9 & 7 & 0 \\
\hline & Ethanol & 0 & - & 0 & 0 & 6 \\
\hline & Galactose & 0 & 0 & 0 & 1 & 1 \\
\hline \multirow{3}{*}{$\begin{array}{l}\text { S. cerevisiae } \mathrm{FH} 4 \mathrm{C}[\mathrm{pFX} 7-\mathrm{N}] \\
\text { YNB-M medium, } \mathrm{pH} \text { control } \\
\text { with } \mathrm{NH}_{4} \mathrm{OH}\end{array}$} & Glucose \& Maltose & 3 & 317 & 10 & 6 & 0 \\
\hline & Ethanol & 0 & - & 0 & 0 & 6 \\
\hline & Galactose & 0 & 0 & 0 & 0 & 1 \\
\hline \multirow{3}{*}{$\begin{array}{l}\text { S. cerevisiae FH4C (wild type) } \\
\text { YNB-M medium, pH control } \\
\text { with } \mathrm{NH}_{4} \mathrm{OH}\end{array}$} & Glucose \& Maltose & 4 & 317 & 11 & 10 & 0 \\
\hline & Ethanol & 0 & - & 0 & 0 & 5 \\
\hline & Galactose & 4 & 66 & 0 & 56 & 2 \\
\hline
\end{tabular}

For a detailed description of growth parameters, culture media and employed vectors, see Antoniukas et al. 2006

\section{Succinate}

Succinate formation was observed only when the YNB-M medium supplemented with malt extract was employed. In contrast to the other products which exhibited sharp peak-shaped excretion and uptake kinetics, succinate was found to increase in parallel with growth, not only during the glucose growth phase but also during growth with ethanol and galactose, respectively. Generally, succinate represents a major fermentation product in S. cerevisiae when a reductively driven tricarboxylic acid cycle (TCA) is activated under anaerobic conditions with fumarate reductase as key enzyme. Since in our cultures the formation of succinate also occurred in the ethanol and galactose growth phases, i.e., under respiratory conditions, we can rule out fumarate reductase to be a metabolic source of succinate. An alternative source for succinate formation in yeast has been reported to originate from the oxidative decarboxylation of 2-oxoglutarate (Camarasa et al. 2003), when cultivated in media containing glutamate. However, in our experiments, succinate excretion was dependent on the presence of malt extract, which is only a poor source of amino acids. The excretion of succinate might thus be an indication of reduced fluxes in the tricarboxylic acid cycle, probably at the succinate dehydrogenase level or subsequent anabolic reactions, which leads to an overflow of succinate via the oxidative TCA reaction sequence.

Influence of alkali ions employed for $\mathrm{pH}$ control

A further important result of our study is the finding that the nature of alkali, used for $\mathrm{pH}$ control, had a marked effect on the profiles of external metabolites. During initial experiments, we employed $\mathrm{NaOH}$ as $\mathrm{pH}$-corrective agent in YNB cultivations and noticed a strong fermentative metabolism with ethanol, pyruvate and acetaldehyde excreted during growth with glucose and significant amounts of acetate produced during the ethanol growth phase (Table 1). Moreover, the galactose growth phase, which as describes above, produced no metabolites with recombinant strains, now resulted in the formation of ethanol and acetate as well as minor amounts of acetaldehyde and pyruvate. We note here that the $\mathrm{pO}_{2}$ was maintained at $70 \%$ air saturation in all cultivations, thus excluding oxygen limiting conditions as a cause for fermentative metabolism. In contrast, as described above and shown in Table 1, when $\mathrm{NaOH}$ was replaced by $\mathrm{NH}_{4} \mathrm{OH}$, none of these metabolites were excreted by actively hantavirus $\mathrm{N}$ protein 
producing recombinant strains during growth with galactose. The same result was also obtained when $\mathrm{KOH}$ was used instead of $\mathrm{NaOH}$ (data not shown). It is known that $S$. cerevisiae does not need sodium ions for growth and that high concentrations are toxic for yeast (Rios et al. 1997). In our case, $260 \mathrm{ml} 1 \mathrm{M}$ $\mathrm{NaOH}$ were added at the end of the cultivation, which resulted in at least $200 \mathrm{mM} \mathrm{Na}{ }^{+}$ions in the culture medium. That sodium ions cause an increase of the metabolic flux through the glycolytic pathway has been described by Olz et al. (1993). This effect can be explained straightforward with a previously described $\mathrm{Na}^{+} / \mathrm{H}^{+}$antiporter system (Prior et al. 1996), which at the expense of cellular ATP, pumps sodium out of the cell. Thus, the resulting drop of the cellular ATP/ADP ratio causes an enhancement of the glycolytic flux by relieving the inhibition of enzymes such as hexokinase and phosphofructokinase, both are regulated allosterically by these nucleotides (Larsson et al. 1997).

In conclusion, and in contrast to a recent report of Ferreira et al. (2004) for the recombinant production of cutinase, we did not find that formation of acetate (or any other metabolite) is related to the onset of the biosynthesis of hantavirus $\mathrm{N}$ protein. In our case the absence of excreted metabolites from the culture broth (except succinate) was specifically associated with the phase of recombinant gene expression. Conversely, the extracellular accumulation of acetate and pyruvate in YNB-M during the galactose phase indicated a non-producing situation. Therefore, the galactose-induced hantavirus $\mathrm{N}$ protein production phase, was characterized by the following parameters which can be routinely obtained from bioreactor cultures: (i) no production of extracellular acetate, accessible online by measuring the amount of added base for $\mathrm{pH}$ control (note that succinate concentrations were ten times lower than acetate (Fig. 1)), and (ii) a low RQ value, comparable to the preceding ethanol growth phase, whereas non-producing cells exhibited a significant increase in the RQ during growth with galactose. As a further result, $\mathrm{pH}$ control should avoid $\mathrm{NaOH}$ due to the inhibiting effect of excess sodium ions on the oxidative metabolism of the employed yeast cells.

The results of this study demonstrate physiological responses of the recombinant host strain to cultivation conditions and expression vector, therefore provides significant information for the further development of an industrial process for hantavirus antigen production.

\section{References}

Allison DG, Sattenstall MA (2007) The influence of green fluorescent protein incorporation on bacterial physiology: a note of caution. J Appl Microbiol 103:318-324

Antoniukas L, Grammel H, Reichl U (2006) Production of hantavirus Puumala nucleocapsid protein in Saccharomyces cerevisiae for vaccine and diagnostics. J Biotechnol 124:347-362

Camarasa C, Grivet JP, Dequin S (2003) Investigation by 13CNMR and tricarboxylic acid (TCA) deletion mutant analysis of pathways for succinate formation in Saccharomyces cerevisiae during anaerobic fermentation. Microbiology 149:2669-2678

Dargeviciute A, Sjolander BK, Sasnauskas K et al (2002) Yeast-expressed Puumala hantavirus nucleocapsid protein induces protection in a bank vole model. Vaccine 20:3523-3531

Ferreira BS, Calado CR, van Keulen F et al (2004) Recombinant Saccharomyces cerevisiae strain triggers acetate production to fuel biosynthetic pathways. J Biotechnol 109:159-167

Janes M, Meyhack B, Zimmerman W, Hinnen A (1990) The influence of GAP promoter variants on hirudin production, average plasmid copy number and cell growth in S. cerevisiae. Curr Genet 18:97-103

Koch J, Brockmann S, Winter C, Kimmig P, Stark K (2007) Significant increase of hantavirus infections in Germany since the beginning of 2007. Euro Surveill 12:E070503.1. http://eurosurveillance.org.asp\#1

Larsson C, Nilsson A, Blomberg A, Gustafsson L (1997) Glycolytic flux is conditionally correlated with ATP concentration in Saccharomyces cerevisiae: a chemostat study under carbon- or nitrogen-limiting conditions. J Bacteriol 179:7243-7250

Olz R, Larsson K, Adler L, Gustafsson L (1993) Energy flux and osmoregulation of Saccharomyces cerevisiae grown in chemostats under $\mathrm{NaCl}$ stress. J Bacteriol 175:22052213

Prior C, Potier S, Souciet JL, Sychrova H (1996) Characterization of the NHA1 gene encoding a $\mathrm{Na}+\mathrm{H}+$-antiporter of the yeast Saccharomyces cerevisiae. FEBS Lett 387:89-93

Rios G, Ferrando A, Serrano R (1997) Mechanisms of salt tolerance conferred by overexpression of the HAL1 gene in Saccharomyces cerevisiae. Yeast 13:515-528

Sasnauskas K, Buzaite O, Vogel F et al (1999) Yeast cells allow high-level expression and formation of polyomavirus-like particles. Biol Chem 380:381-386

Snoep JL, Yomano LP, Westerhoff HV, Ingram LO (1995) Protein burden in Zymomonas mobilis: negative flux and growth control due to overproduction of glycolytic enzymes. Microbiology 141:2329-2337 